\title{
Géolinguistique
}

$21 \mid 2021$

Varia

\section{Toponymes et parlers régionaux de la région de Toyama : autour des noms dialectaux de la pomme de terre dans les bassins des rivières Jinzū et Shō}

Toponyms and Dialects in Toyama Prefecture: A Focus on the Dialectal Names of Potatoes in the Jinzū and Shō River Basins

\section{Seiichi Nakai}

Traducteur : Mailys Leeman

\section{OpenEdition}

\section{Journals}

Édition électronique

URL : https://journals.openedition.org/geolinguistique/5915

DOI : 10.4000/geolinguistique.5915

ISSN : 2650-8176

Éditeur

UGA Éditions/Université Grenoble Alpes

Édition imprimée

ISBN : 978-2-37747-303-8

ISSN : 0761-9081

Référence électronique

Seiichi Nakai, «Toponymes et parlers régionaux de la région de Toyama : autour des noms dialectaux de la pomme de terre dans les bassins des rivières Jinzū et Shō », Géolinguistique [En ligne], 21 | 2021, mis en ligne le 06 décembre 2021, consulté le 19 décembre 2021. URL : http://

journals.openedition.org/geolinguistique/5915; DOI : https://doi.org/10.4000/geolinguistique.5915

Ce document a été généré automatiquement le 19 décembre 2021.

Géolinguistique 


\section{Toponymes et parlers régionaux de la région de Toyama : autour des noms dialectaux de la pomme de terre dans les bassins des rivières Jinzū et Shō}

Toponyms and Dialects in Toyama Prefecture: A Focus on the Dialectal Names of Potatoes in the Jinzū and Shō River Basins

\section{Seiichi Nakai}

Traduction : Mailys Leeman

\section{NOTE DE L'AUTEUR}

Bien que le système de translittération Kunrei figure initialement sur les différentes illustrations que comprend cet article, le système de translittération Hepburn, plus fidèle à la phonétique du japonais, a été préféré dans le corps du texte. Les parties entre crochets correspondent à des ajouts explicatifs. Enfin, les personnes mentionnées dans l'article le sont par leur nom de famille suivi de leur prénom.

Nous remercions Mailys Leeman, étudiante en master Traduction spécialisée multilingue à l'université Grenoble Alpes, pour la traduction de cet article.

\section{Introduction}

En linguistique, pourquoi le phénomène de différenciation diatopique se produit-il? Quels sont, en arrière-plan, les divers facteurs qui contribuent à la création des formes dialectales? Ils se profilent comme étant complexes et interdépendants. La manière la 
plus appropriée d'aborder le caractère universel et singulier dans la formation des unités lexicales revient donc à se concentrer sur ceux dont la période d'apparition dans le langage est connue.

La patate douce, la pomme de terre ou encore le piment sont autant de variétés exotiques dont la date d'introduction du vocable est connue. En effet, elles ont été importées au Japon entre le $\mathrm{XVI}^{\mathrm{e}}$ et le $\mathrm{XVII}^{\mathrm{e}}$ siècle. Les deux premières arborent une multitude de noms dialectaux, certains liés à des toponymes et dont il est généralement admis qu'ils corrèlent avec l'itinéraire par lequel ces variétés ont été introduites: Ryūkyū, Satsuma, Sendai, Kōshū...

Selon Sawaki (1979), « la dénomination de toute chose nouvellement importée peut se faire de deux façons principales: en lui appliquant un nouveau terme (création lexicale) ou bien en s'appropriant le nom déjà existant dans le lieu d'origine d'où elle a été exportée ».

Nous baserons notre analyse des différents noms dialectaux de la pomme de terre sur le Nihon gengo chizu (Linguistic Atlas of Japan, LAJ), élaboré entre 1957 et 1965, ainsi que sur le Field Research Project to Analyze the Formation Process of Japanese Dialects (FPJD) conduit de 2010 à 2015.

Les résultats recueillis aux fins du LAJ ont été publiés en six volumes entre 1966 et 1974 par le Kokuritsu kokugo kenkyūjo (National Institute for Japanese Language and Linguistics, NINJAL), au terme d'enquêtes de terrain. Au total, 285 entrées avaient été couvertes au travers de 2400 points d'enquête. Le FPJD, un projet d'ampleur nationale visant à analyser le procédé de formation des dialectes, a été mené plusieurs décennies plus tard par le NINJAL, sous la direction d'Ōnishi Takuichirō, dans 554 points d'enquête. Le Shin Nihon gengo chizu (New Linguistic Atlas of Japan, NLJ) regroupe les résultats de ces enquêtes collectives.

6 Nous observerons également la distribution géographique des données linguistiques afin de déterminer en quoi celle-ci a pu être conditionnée par la topographie de la préfecture de Toyama, avec ses deux principaux cours d'eau que sont les rivières Jinzū et Shō. À cette fin, nous nous appuierons sur les données d'enquêtes conduites respectivement de 2009 à 2011 sur le bassin de la rivière Jinzū, et de 2009 à 2013 sur celui de la rivière Shō. La seconde a été menée dans 176 points d'enquête : depuis le village de Shirakawa dans la préfecture de Gifu, en amont de la rivière, jusqu'à la ville de Shinminato (l'actuelle Imizu) située au bord de la baie de Toyama. Chaque kōminkan (centre communautaire d'apprentissage) a apporté son soutien au projet. Enfin, la première a été réalisée dans 159 points d'enquête : depuis l'ancien village de Hosoiri situé dans la préfecture de Toyama, près de la frontière avec celle de Gifu, jusqu'à Iwase dans la ville de Toyama, au bord de la baie. La ville de Toyama a soutenu l'ensemble du projet.

\section{Relation entre toponymes et noms botaniques}

\subsection{Noms dialectaux de la patate douce (satsumaimo ${ }^{1}$ )}


Carte A. - Répartition des noms dialectaux de la patate douce dans tout le Japon.

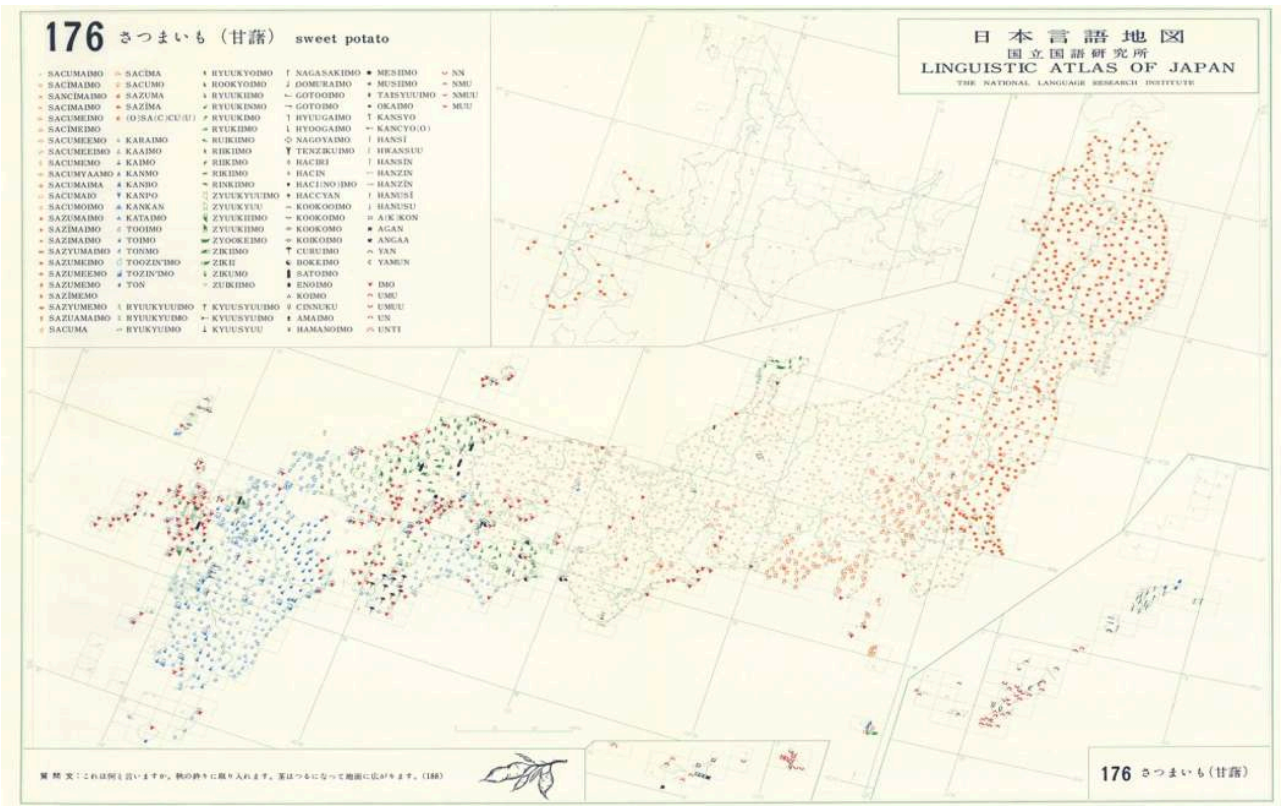

Tirée du Linguistic Atlas of Japan : <https://mmsrv.ninjal.ac.jp/laj_map/data/LAJ_176.html>.

On estime que c'est en l'an 1597, sur l'île de Miyako, que la patate douce a été introduite pour la première fois au Japon. Transportée jusqu'à Satsuma et Nagasaki au début du $\mathrm{XVII}^{\mathrm{e}}$ siècle, sa culture s'est progressivement propagée dans tout le sud de l'île de Kyūshū. En raison de son bon rendement même sur sol aride et les années de mauvaises récoltes pour les autres cultures vivrières, elle s'est imposée comme aliment important pour lutter contre la famine durant les fréquents épisodes de pénurie de l'époque. Elle sera ainsi introduite au xviII ${ }^{e}$ siècle à Edo (l'actuelle Tōkyō) par l'érudit Aoki Kon'yō. Sa transmission se poursuivra de manière proactive aux autres régions, à mesure que les famines s'abattront sur les populations. À la fin de l'ère Edo (1603-1868), sa culture se sera étendue jusqu'à la région du Tōhoku.

Les noms dialectaux de la patate douce vont de pair avec les toponymes des présumés lieux depuis lesquels elle a été introduite: la forme satsumaimo s'observe depuis la région Est du Japon jusqu'aux régions du Kinki et du Chūgoku à l'ouest. Celle de tōimo du nord de l'île de Kyūshū jusqu'aux alentours de la préfecture de Yamaguchi. La forme karaimo se retrouve dans la partie sud de l'île de Kyūshū et dans certaines parties de l'île de Shikoku, tandis que ryūkyūimo se rencontre entre autres dans le nord-ouest de l'île de Kyūshū, dans la région du Chūgoku, sur l'île de Shikoku et sur la péninsule de Noto.

Les principaux noms vernaculaires de la patate douce se répartissent ainsi d'ouest en est comme suit : karaimo $\rightarrow$ töimo $\rightarrow$ ryūkyūimo $\rightarrow$ satsumaimo. Cet ordre correspond à des noms de lieux, du plus au moins éloigné de l'intérieur du Japon. En effet, lorsque la patate douce a été introduite dans l'archipel des Ryūkyū et dans le sud de l'île de Kyūshū, elle a été dénommée kara-imo ou tō-imo ('tubercule provenant de l'étranger'), puis ryūkyū-imo ('tubercule provenant des Ryūkyū') lorsqu'elle a été transportée à une certaine distance de l'archipel des Ryūkyū, et enfin satsuma-imo ('tubercule provenant de Satsuma') lors de son introduction à Edo depuis cette province. 


\subsection{Noms dialectaux de la pomme de terre $\left(\right.$ jagaimo $\left.^{2}\right)$}

Carte B. - Répartition des noms dialectaux prévalents de la pomme de terre dans tout le Japon.

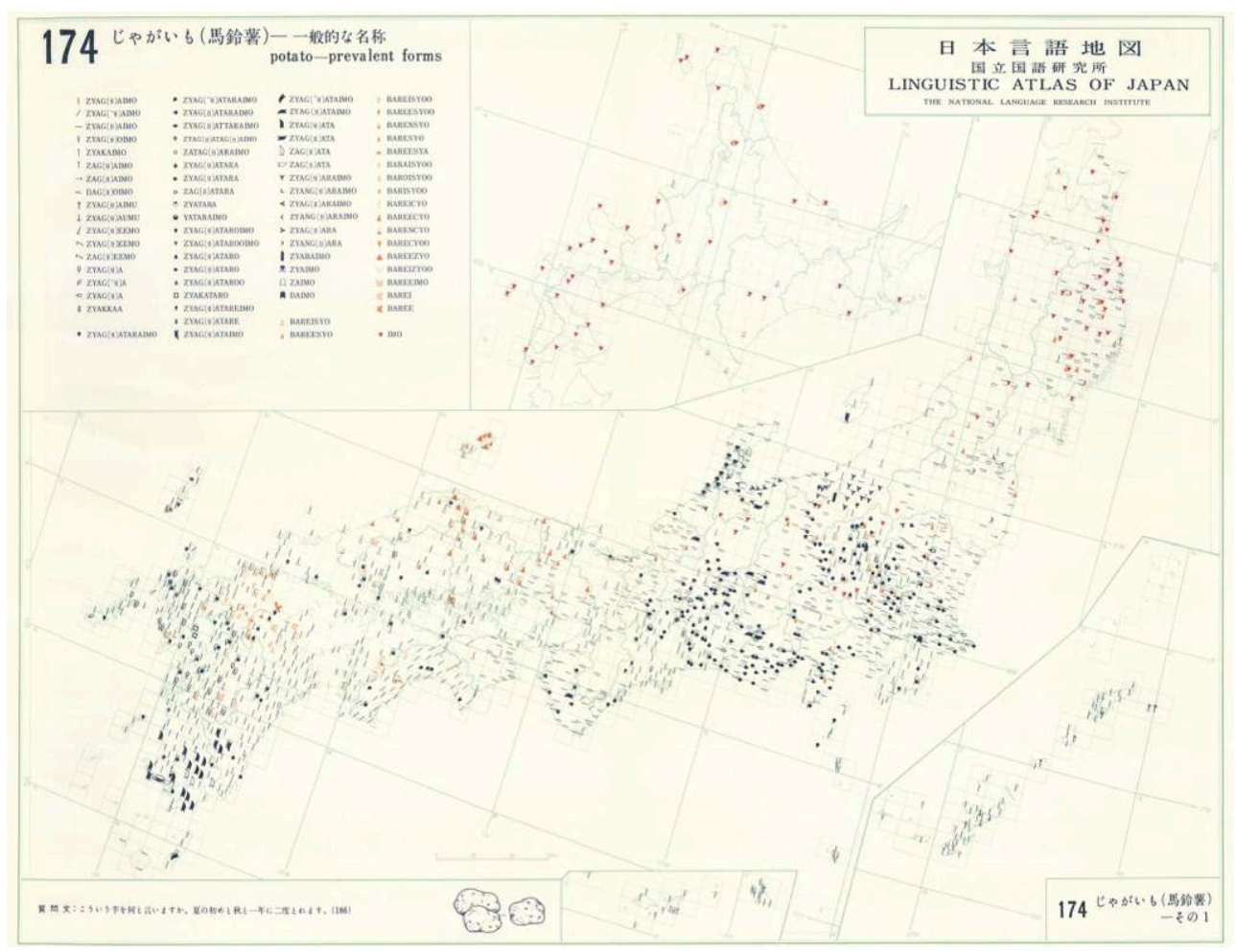

Tirée du Linguistic Atlas of Japan : <https://mmsrv.ninjal.ac.jp/laj_map/data/LAJ_174.html>.

10 Le LAJ présente deux cartes relatives à la répartition des noms dialectaux de la pomme de terre: l'une sur les noms prévalents (ci-dessus) et l'autre sur les noms spécifiques. D'après la carte $\mathrm{B}$, la forme imo se retrouve sur l'île de Hokkaidō ainsi que dans la région du Tōhoku, tandis que bareisho, représentée par les symboles de couleur orange, est commune dans certaines parties de la région du Kantō, dans la région du Chūgoku ainsi que dans la partie nord de l'île de Kyūshū. Globalement, la forme jagaimo prédomine.

11 La pomme de terre a été introduite pour la première fois au Japon en l'an 1601, par le biais d'un navire hollandais naviguant depuis le port de Jakarta (anciennement Jakatora en japonais) jusqu'à la ville portuaire de Hirado, dans la préfecture de Nagasaki. L'appellation jagataraimo ${ }^{3}$ lui a été attribuée, abrégée plus tard en jagaimo. Pendant l'ère Kansei (1789-1801), la pomme de terre a cette fois été introduite sur l'île de Hokkaidō depuis l'empire de Russie, et s'est répandue dans la région du Tōhoku sous la forme ezoimo ${ }^{4}$. Comme en Europe, cette variété a d'abord été utilisée au Japon comme plante ornementale, puis s'est révélée précieuse lors des périodes récurrentes de famine. Sa culture se répandra ainsi comme aliment de famine dans tout le pays avant la fin de l'ère Edo, au milieu du xix siècle. Mais ce n'est qu'au début de l'ère Meiji (1868-1912), après qu'une variété de qualité supérieure aura notamment été importée des États-Unis par la Hokkaidō kaitakushi (Mission au défrichement de Hokkaidō), que la culture de la pomme de terre se généralisera réellement dans l'archipel. 
Carte C. - Répartition des noms dialectaux spécifiques de la pomme de terre dans tout le Japon.

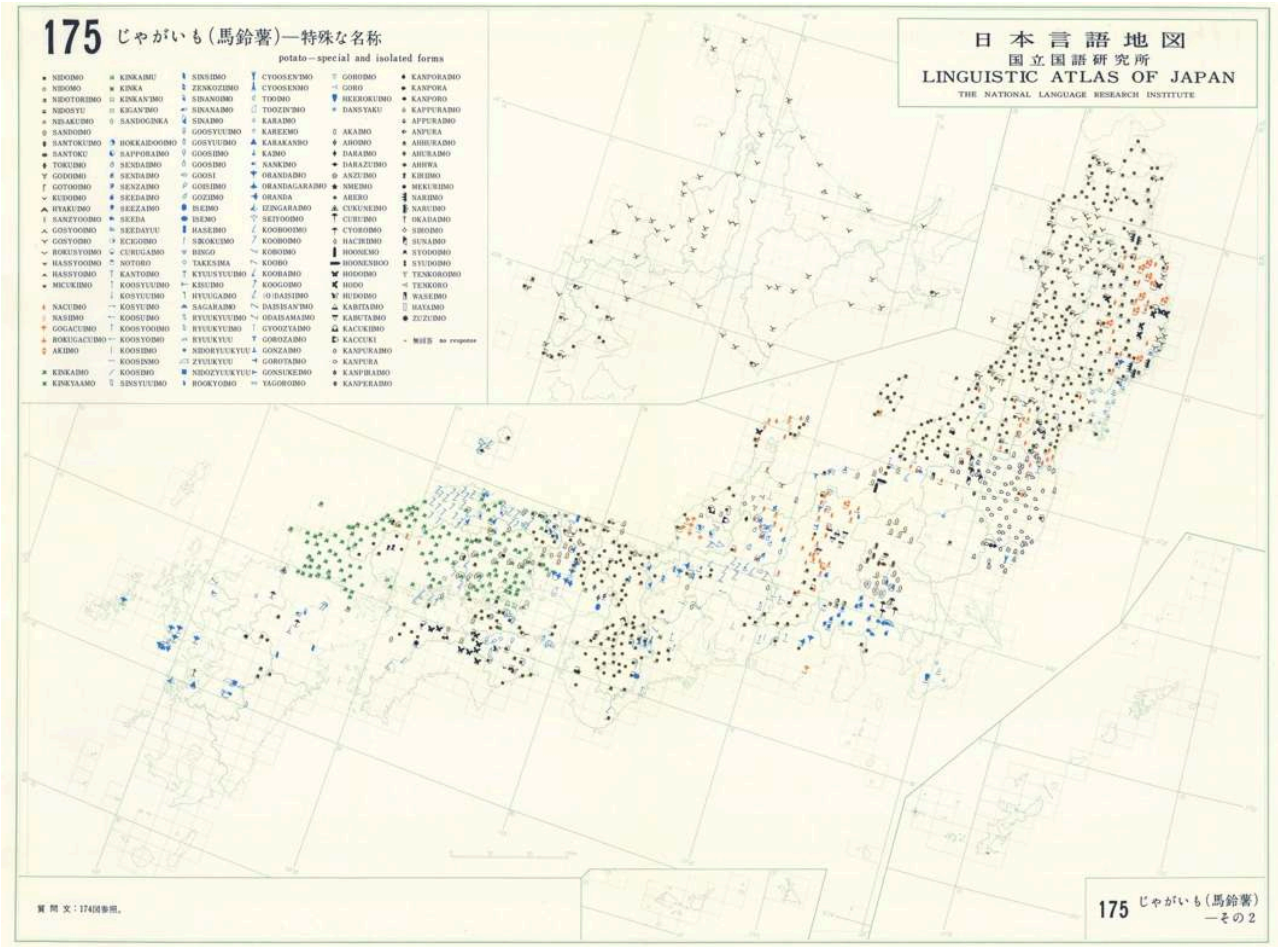

Tirée du Linguistic Atlas of Japan : <https://mmsrv.ninjal.ac.jp/laj_map/data/LAJ_175.html>.

Les noms vernaculaires de la pomme de terre sont très souvent liés à des toponymes japonais : par exemple, hokkaidōimo dans les préfectures de Tottori et Fukui, sendaiimo dans celle de Gifu, echigoimo à Shiga, tsurugaimo à Iwate, kōshūimo dans la région du Tōkai notamment, shinshūimo à Saitama, gōshūimo à Tokushima, iseimo à Hyōgo, shikokuimo à Niigata et Hyōgo, hyūgaimo à Nara, ou encore ryūkyūimo aux alentours de Kumamoto. Chaque forme d'inspiration toponymique est employée loin de la région qui donne son nom à la pomme de terre.

La forme nidoimo provient du fait que ce tubercule peut être récolté deux fois par an dans certaines régions : nido signifie ici 'deux fois'. On pense ainsi que les formes sandoimo et godoimo pourraient en être des formes exagérées: chaque préfixe signifierait respectivement 'trois fois' et 'cinq fois'.

La forme goshoimo, prédominante sur l'île de Hokkaidō, se rapporte au grand rendement de la pomme de terre : go signifie ici 'cinq' et sho est une unité de mesure du système shakkan ( 1 sho équivaut à environ $1,8 \mathrm{~L}$ ). À ce propos, une hypothèse est avancée selon laquelle la forme godoimo, citée ci-dessus, résulte plutôt d'une vocalisation de la consonne intervocalique de gotoimo (1 to équivaut à environ $18 \mathrm{~L}$ ). Quant à l'origine de la forme sēdaimo, on l'attribue à Nakai Seidayū, magistrat de la ville de Kōfu qui a énormément contribué à répandre la culture de la pomme de terre à la fin du XVII ${ }^{\mathrm{e}}$ siècle. 
Carte D. - Répartition des noms dialectaux spécifiques de la pomme de terre : simplification $n^{\circ} 1$.

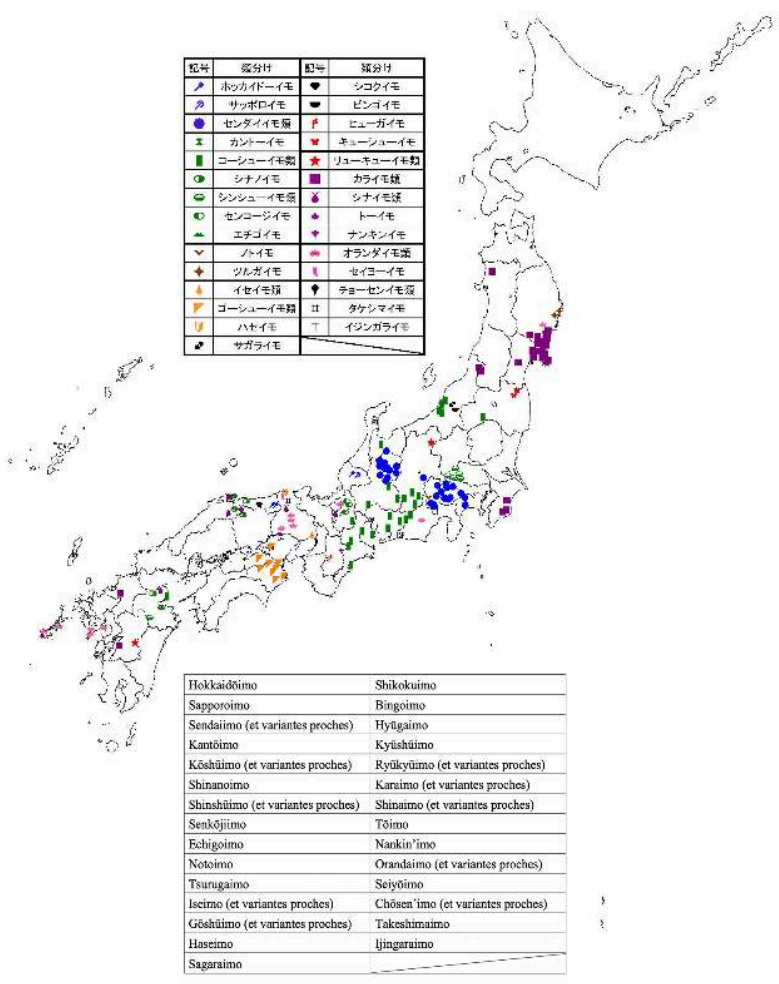

Tirée du Linguistic Atlas of Japan.

Carte E. - Répartition des noms dialectaux spécifiques de la pomme de terre : simplification $n^{\circ} 2$.

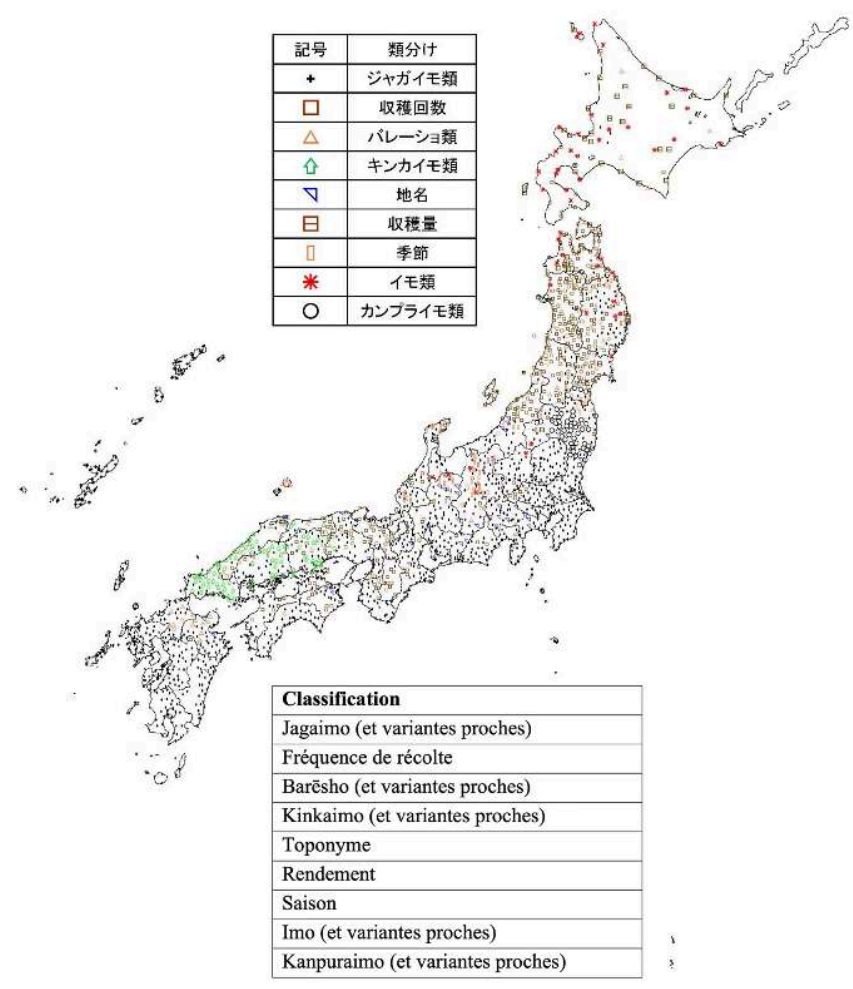

Tirée du Linguistic Atlas of Japan. 
Carte F. - Signification de la forme imo.

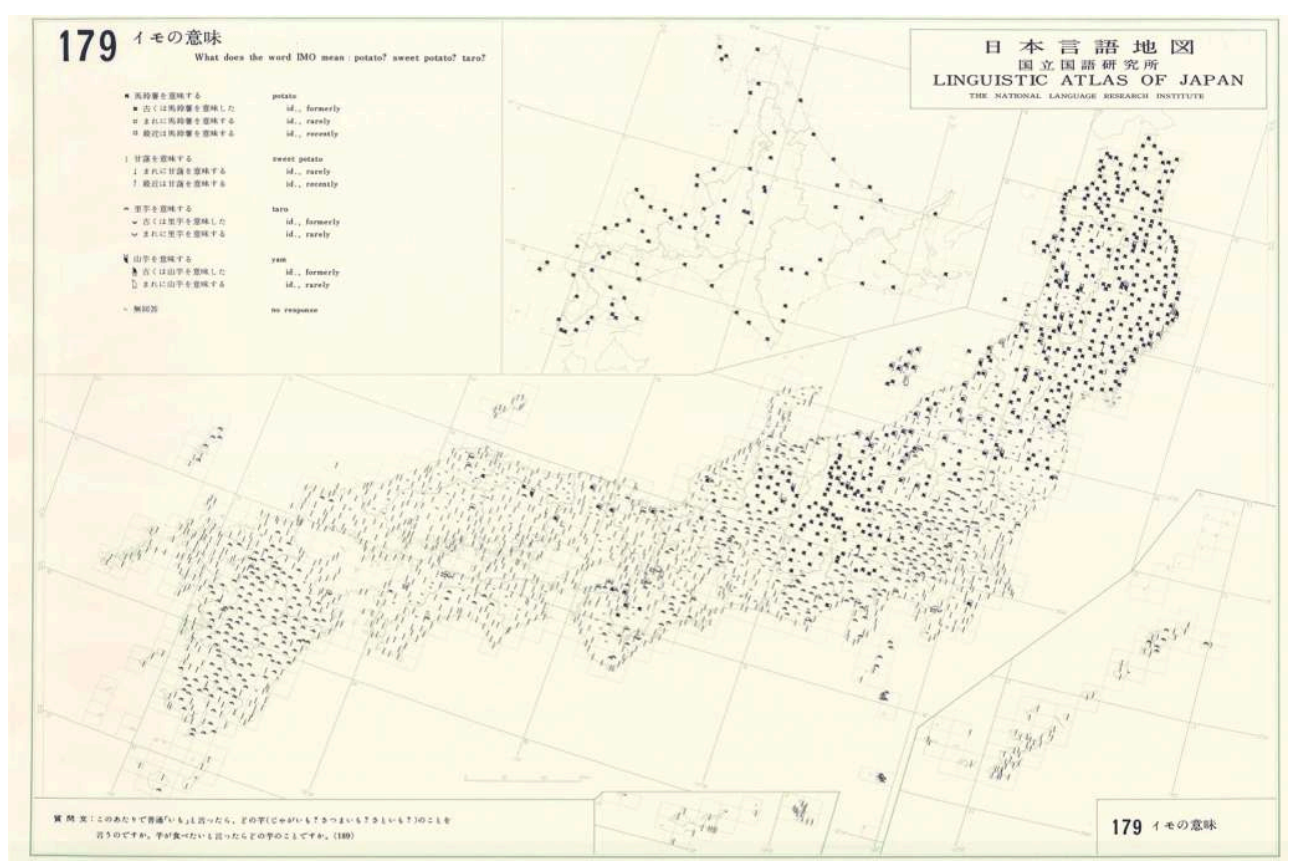

Tirée du Linguistic Atlas of Japan : <https://mmsrv.ninjal.ac.jp/laj_map/data/LAJ_179.html>.

Le LAJ recense également les types de tubercules auxquels la forme imo fait référence. Les réponses obtenues semblent correspondre aux variétés les plus couramment cultivées dans chaque région. Cette forme se rapporte à la pomme de terre dans la partie nord de la région du Chūbu, tandis qu'elle se rapporte à la patate douce dans de nombreuses autres régions. Le taro est largement mentionné de la région du Kantō à celles du Tōkai et du Hokuriku, ainsi que sur l'île de Kyūshū. C'est dans ces mêmes régions que la culture du taro était prospère. Il porte également des appellations telles que hon'imo ou encore maimo (ces deux préfixes signifient 'authentique'), témoignant de l'idée d'un tubercule originel par opposition à la pomme de terre et à la patate douce, toutes deux introduites depuis l'étranger. La forme yamaimo ('tubercule de montagne') est par ailleurs attribuée dans les préfectures d'Akita et d'Iwate notamment.

Tableau A. - Types de tubercules se rapportant à une appellation donnée.

\begin{tabular}{|l|l|l|l|l|}
\hline & Taro & Patate douce & Pomme de terre & Total \\
\hline akaimo & & & & 2 \\
\hline igaimo & & & & 2 \\
\hline imo & & & & 3 \\
\hline imonoko & & & & 2 \\
\hline emo & & & & 2 \\
\hline
\end{tabular}




\begin{tabular}{|l|l|l|l|l|}
\hline okaimo & & & & 2 \\
\hline orandaimo & & & & 2 \\
\hline kaimo & & & & 3 \\
\hline karaimo & & & & 2 \\
\hline genkiimo & & & & 3 \\
\hline jagaimo & & & & 2 \\
\hline shiroimo & & & & 2 \\
\hline zuikiimo & & & & 2 \\
\hline tsuruimo & & & & 2 \\
\hline tenjikuimo & & & & 2 \\
\hline toimo & & & & 2 \\
\hline tōimo & & & & 2 \\
\hline tonoimo & & & & 2 \\
\hline nankin'imo & & & & 2 \\
\hline baraimo & & & & 2 \\
\hline hyūgaimo & & & & 2 \\
\hline maimo & & & & \\
\hline ryūimo & & & & 2 \\
\hline
\end{tabular}

17 Nous remarquons ainsi l'existence d'homonymes parmi les nombreux noms dialectaux de la patate douce, de la pomme de terre et du taro. 
Carte G. - Signification de la forme imo.

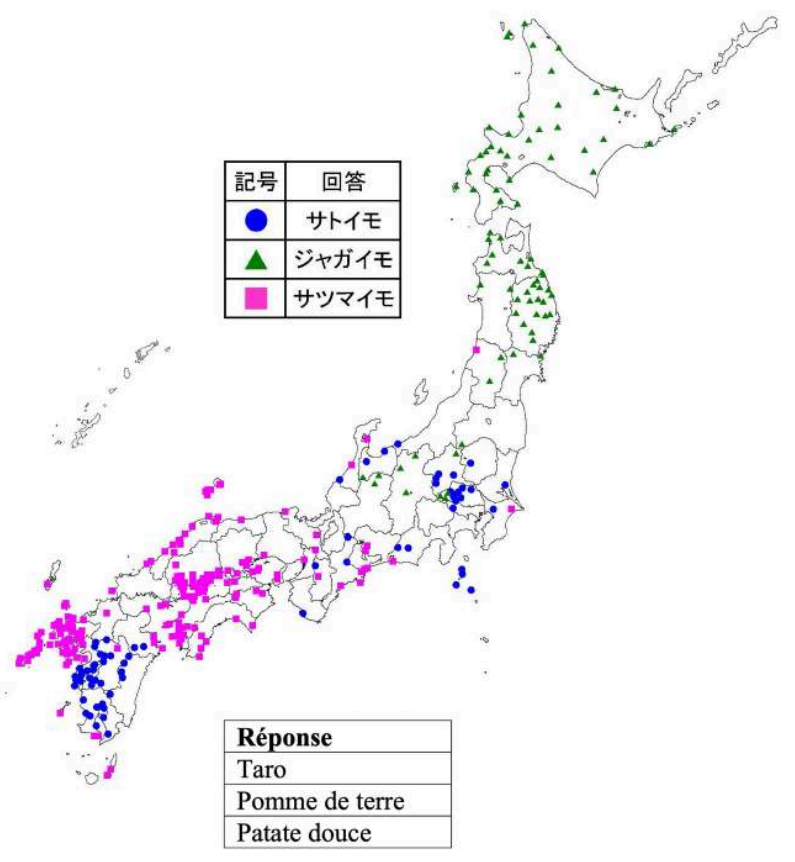

Tirée du Linguistic Atlas of Japan.

Carte H. - Signification de la forme tōimo.

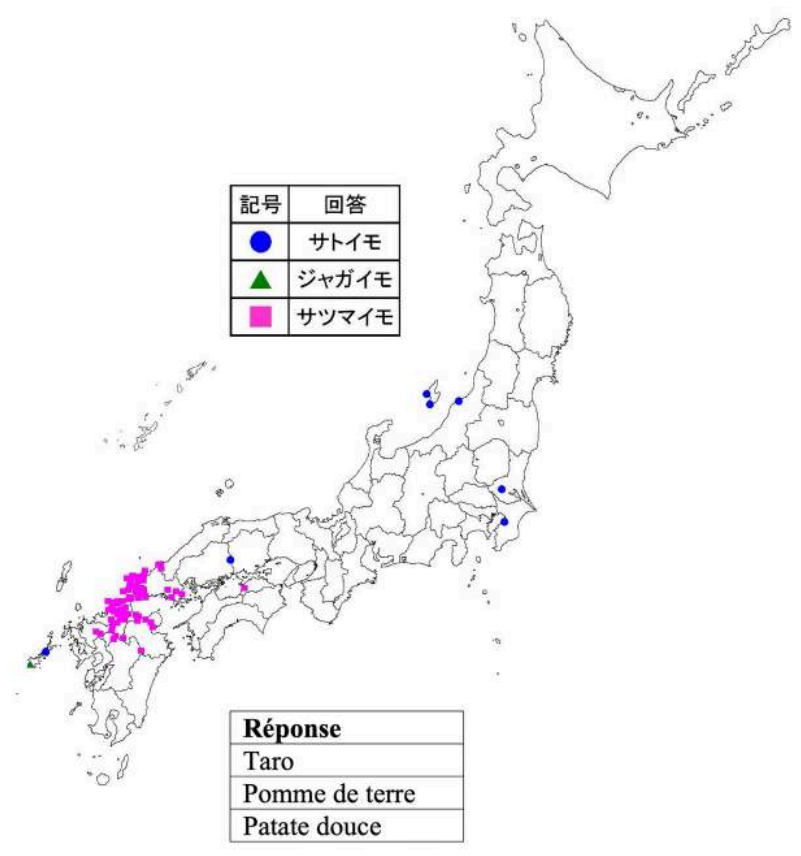

Tirée du Linguistic Atlas of Japan. 
En fonction de la région, la forme akaimo se réfère soit au taro, soit à la pomme de terre, alors que d'autres formes telles qu'imo et tōimo se réfèrent à chacun des trois types de tubercules.

Carte I. - Signification de la forme karaimo.

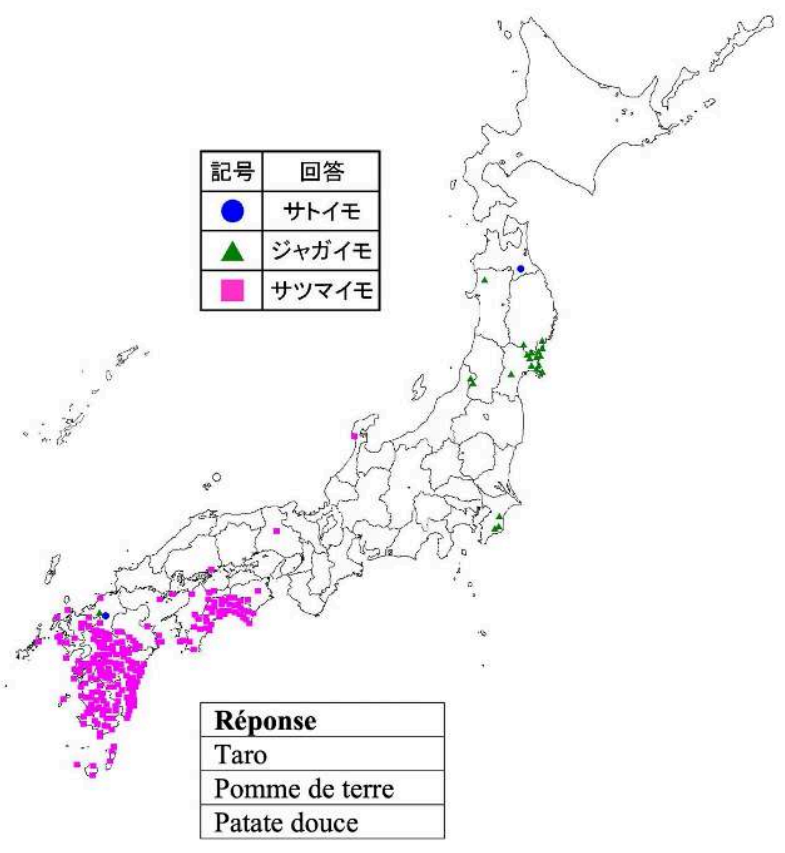

Tirée du Linguistic Atlas of Japan.

19 Analysons de plus près encore ces collisions homonymiques. La forme imo se réfère à la patate douce depuis la partie nord de l'île de Kyūshū jusqu'aux régions de Setouchi, du Kinki, du Tōkai et du Hokuriku. Elle se réfère à la pomme de terre dans la région du Tōhoku principalement, ainsi que dans les préfectures de Gifu, de Nagano et dans la région du Kantō, alors qu'elle se réfère au taro dans les parties centrale et sud de l'île de Kyūshū ainsi que dans la région du Kantō et sa périphérie.

La forme töimo se réfère à la patate douce notamment dans la partie nord de l'île de Kyūshū, à la pomme de terre dans un point d'enquête de la préfecture de Nagasaki, et au taro dans quelques points d'enquête de cette même préfecture ainsi que de celles d'Okayama, d'Ibaraki, de Chiba et de Niigata avec l'île de Sado.

Enfin, la forme karaimo se réfère à la patate douce sur l'île de Kyūshū et plus particulièrement dans sa partie sud, ainsi que sur l'île de Shikoku. Elle se réfère à la pomme de terre dans des préfectures de la région du Tōhoku telles que Miyagi, Iwate et Yamagata, ainsi qu'à Chiba, alors qu'elle se réfère au taro dans quelques localités de la préfecture d'Aomori ou encore de celle de Fukuoka, de sorte que des collisions homonymiques n'ont pas émergé pour ce cas-ci. 


\section{Du LAJ au NLJ : changements de distribution observables dans le FPJD}

Le NINJAL a mené un projet d'enquêtes collectives sous la direction du professeur Ōnishi Takuichirō afin d'analyser le processus de formation des dialectes. De 2010 à 2015, 554 points d'enquête répartis à travers l'archipel ont ainsi fait l'objet de ce projet de collecte d'informations sur le terrain. Les résultats du FPJD, abréviation du nom anglais Field Research Project to Analyze the Formation Process of Japanese Dialects, ont été publiés sous la forme du NLJ par la maison d'édition Asakura Shoten.

Carte J. - Répartition des noms dialectaux de la pomme de terre.
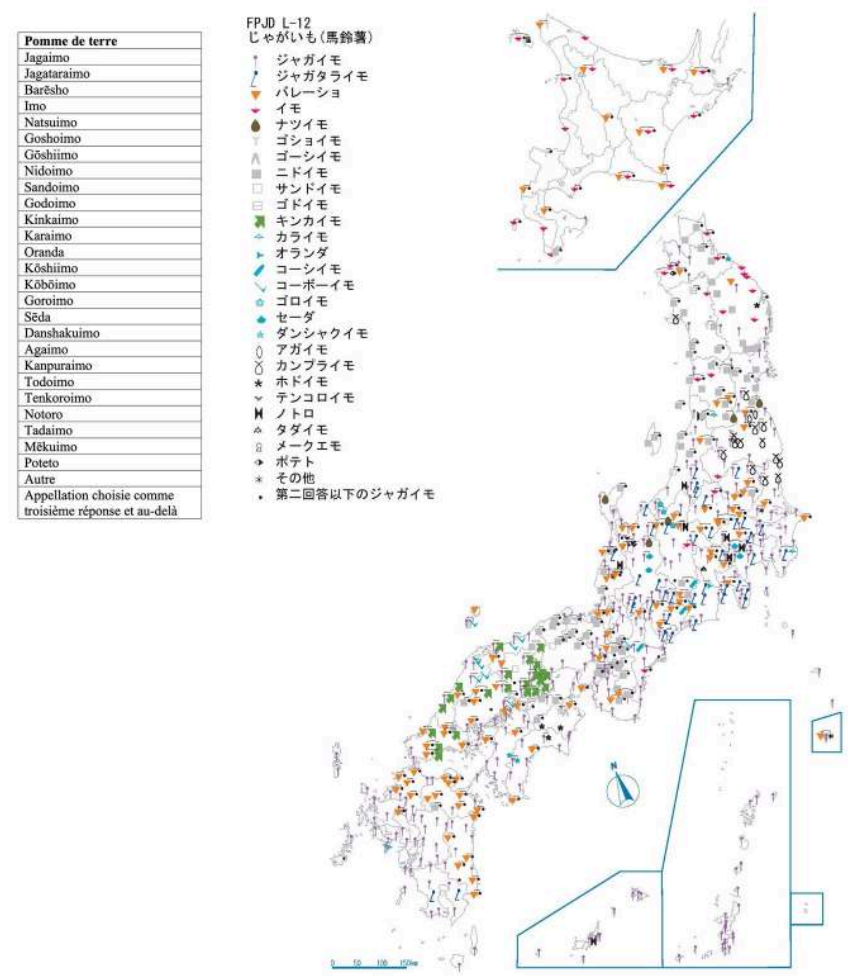

Tirée du New Linguistic Atlas of Japan : <https://www2.ninjal.ac.jp/hogen/dp/fpjd/fpjd_index.html>

L'emploi des noms dialectaux de la pomme de terre a grandement évolué depuis la parution du LAJ. Nous nous concentrerons sur les hyperonymes afin de considérer concrètement ces modifications. Observons dans un premier temps le déplacement de la moyenne des coordonnées géographiques (point central) entre les deux atlas (il est possible de le faire en accédant au site web Quantum GIS). 


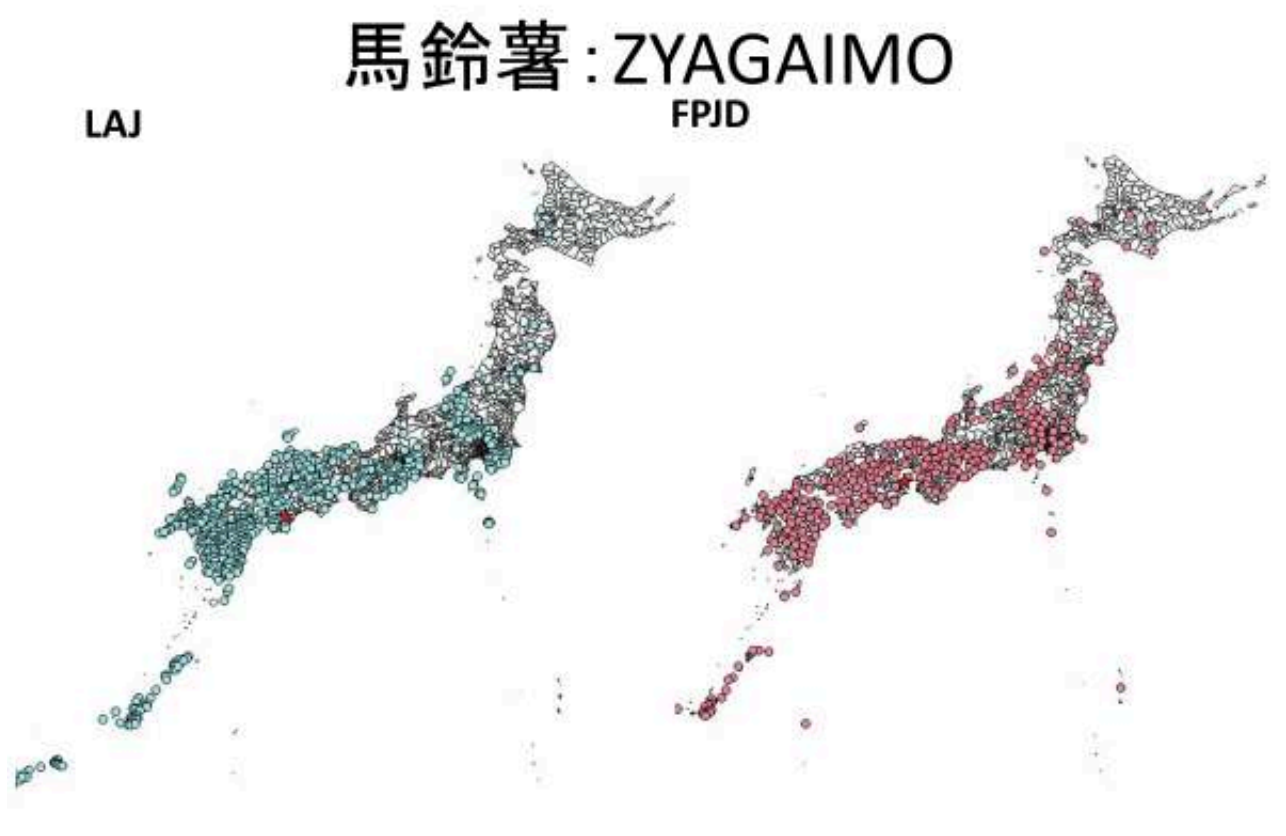

La forme jagaimo, qui est celle du japonais véhiculaire pour désigner la pomme de terre, a vu son utilisation s'étendre de la région du Kinki vers celles du Hokuriku et du Kantō. Son point central (marqué en rouge sur chaque carte) s'est déplacé de manière significative vers l'Est.

Carte L. - Comparaison entre le LAJ et le FPJD : nidoimo.

LAJ

\section{馬鈴薯： NIDOIMO}

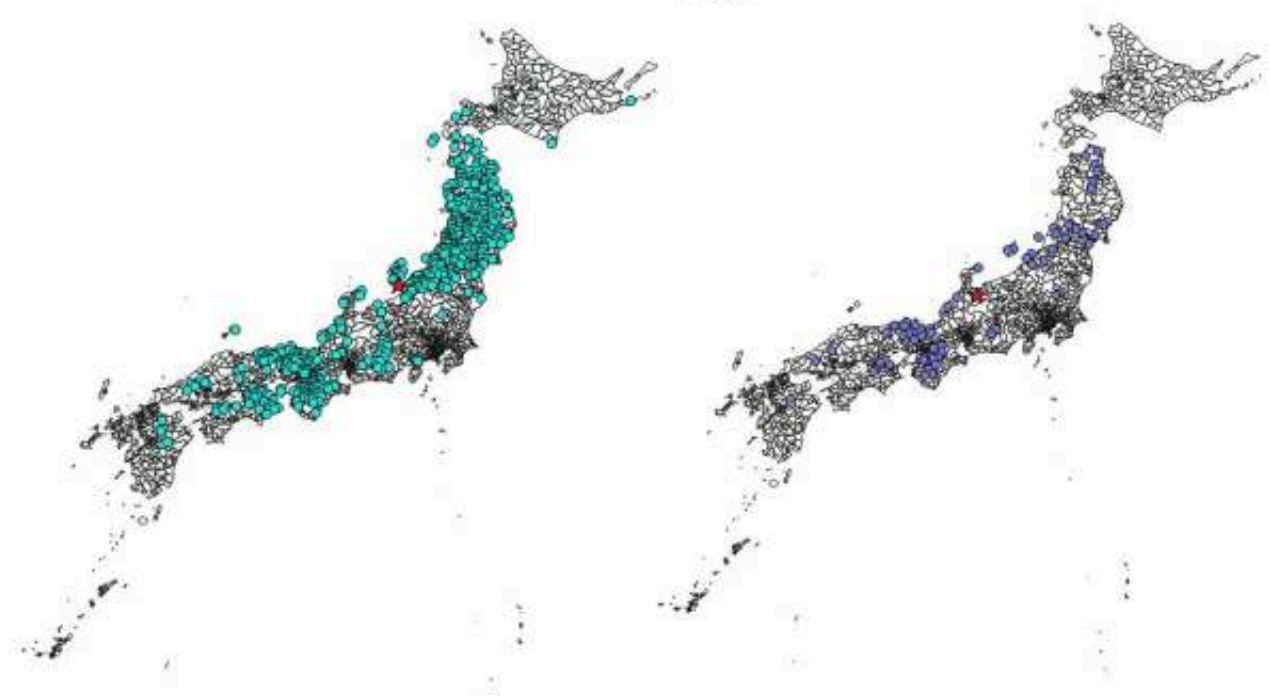


La forme nidoimo 'pomme de terre' provient du fait que cette variété est récoltée deux fois dans l'année. Cette forme est désormais beaucoup moins employée dans tout le pays, notamment dans la région du Tōhoku et dans la préfecture de Niigata. Son point central s'est déplacé vers l'ouest.

Carte M. - Comparaison entre le LAJ et le FPJD : jagatara.

LAJ

\section{馬鈴薯: ZYAGATARA}

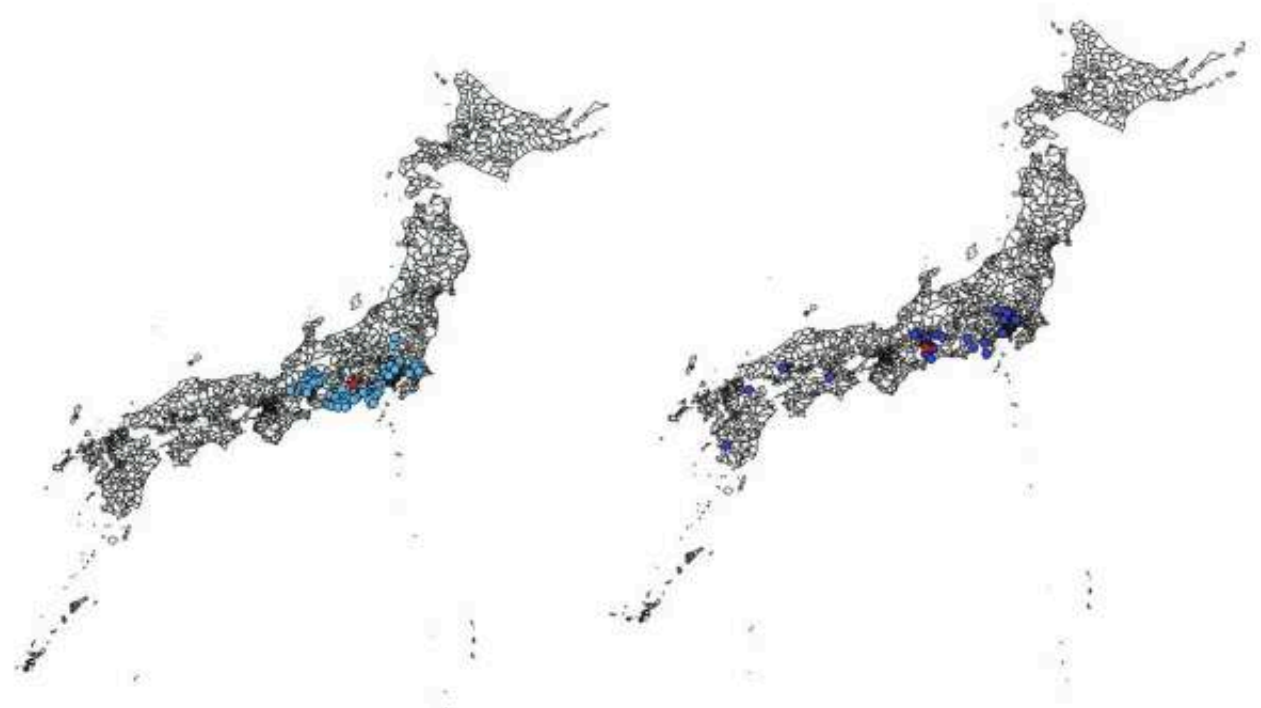

L'emploi de la forme jagatara 'pomme de terre' s'est globalement amoindri. Il subsiste sur l'île de Kyūshū ainsi que dans l'ouest du Japon. Son point central s'est ainsi légèrement déplacé vers l'ouest. 
Carte N. - Comparaison entre le LAJ et le FPJD : bareisho.

\section{馬鈴薯: BAREISYO}

LAJ

FPJD

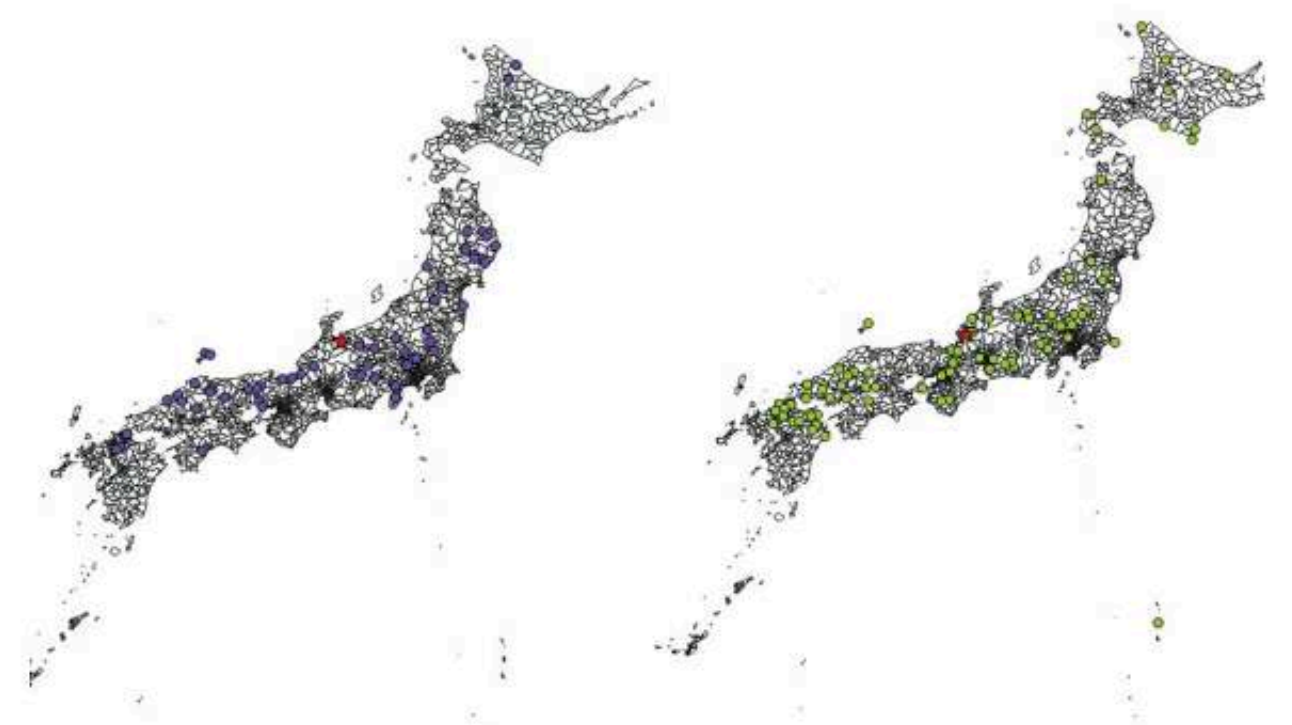

L'emploi de la forme bareisho 'pomme de terre' a augmenté dans tout le pays, étant considérée comme une forme véhiculaire au même titre que jagaimo.

Carte 0. - Comparaison entre le LAJ et le FPJD : kinkaimo.

LAJ

\section{馬鈴薯：KINKAIMO}

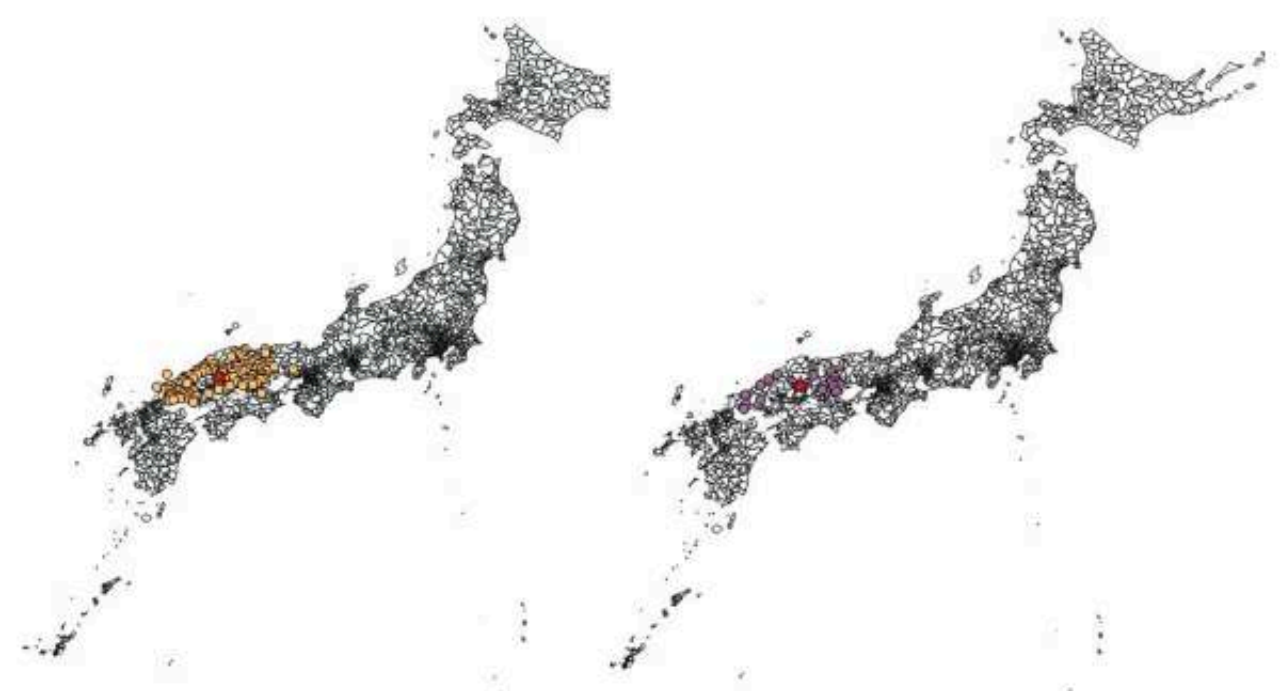


La forme kinkaimo 'pomme de terre' provient de la peau lisse de ce tubercule (qui rappelle celle d'une tête chauve (kinka)) : elle était prédominante dans la région San'inSan'yō mais a fortement reculé. Concentrons-nous maintenant sur la région du Hokuriku (préfecture de Shiga incluse) et observons l'évolution dans le temps de l'emploi des noms dialectaux de la pomme de terre.

Tableau B. - Comparaison entre le LAJ et le FPJD : noms dialectaux de la pomme de terre.

\begin{tabular}{|c|c|c|c|c|c|c|c|c|c|c|}
\hline \multirow[b]{2}{*}{ Nom } & \multicolumn{2}{|c|}{$\begin{array}{c}\text { Préfecture de } \\
\text { Niigata }\end{array}$} & \multicolumn{2}{|c|}{$\begin{array}{c}\text { Préfecture de } \\
\text { Toyama }\end{array}$} & \multicolumn{2}{|c|}{$\begin{array}{l}\text { Préfecture } \\
\text { d'Ishikawa }\end{array}$} & \multicolumn{2}{|c|}{$\begin{array}{l}\text { Préfecture de } \\
\text { Fukui }\end{array}$} & \multicolumn{2}{|c|}{$\begin{array}{c}\text { Préfecture de } \\
\text { Shiga }\end{array}$} \\
\hline & LAJ & FPJD & LAJ & FPJD & LAJ & FPJD & LAJ & FPJD & LAJ & FPJD \\
\hline $\begin{array}{l}\text { Jagaimo } \\
\text { (et variantes } \\
\text { proches) }\end{array}$ & $\begin{array}{c}24 \\
20,51 \%\end{array}$ & $\begin{array}{c}16 \\
51,61 \%\end{array}$ & $\begin{array}{c}11 \\
30,55 \%\end{array}$ & $\begin{array}{c}12 \\
60,00 \%\end{array}$ & $\begin{array}{c}26 \\
48,14 \%\end{array}$ & $\begin{array}{c}10 \\
62,50 \%\end{array}$ & $\begin{array}{c}24 \\
42,85 \%\end{array}$ & $\begin{array}{c}8 \\
53,33 \%\end{array}$ & $\begin{array}{c}20 \\
51,28 \%\end{array}$ & $\begin{array}{c}7 \\
63,63 \%\end{array}$ \\
\hline $\begin{array}{l}\text { Jagataraimo } \\
\text { (et variantes } \\
\text { proches) }\end{array}$ & $\begin{array}{c}16 \\
13,67 \%\end{array}$ & $\begin{array}{c}3 \\
9,67 \%\end{array}$ & $\begin{array}{c}15 \\
41,66 \%\end{array}$ & $\begin{array}{c}2 \\
10,00 \%\end{array}$ & $\begin{array}{c}1 \\
1,85 \%\end{array}$ & $\begin{array}{c}1 \\
6,25 \%\end{array}$ & $\begin{array}{c}3 \\
5,35 \%\end{array}$ & & $\begin{array}{c}4 \\
10,25 \%\end{array}$ & $\begin{array}{c}1 \\
9,09 \%\end{array}$ \\
\hline $\begin{array}{l}\text { Barēsho } \\
\text { (et variantes } \\
\text { proches) }\end{array}$ & & $\begin{array}{c}1 \\
3,22 \%\end{array}$ & & $\begin{array}{c}3 \\
15,00 \%\end{array}$ & & $\begin{array}{c}3 \\
6,25 \%\end{array}$ & $\begin{array}{c}4 \\
7,14 \%\end{array}$ & $\begin{array}{c}4 \\
26,66 \%\end{array}$ & $\begin{array}{c}1 \\
2,56 \%\end{array}$ & $\begin{array}{c}2 \\
18,18 \%\end{array}$ \\
\hline $\begin{array}{l}\text { Nidoimo } \\
\text { (et variantes } \\
\text { proches) }\end{array}$ & $\begin{array}{c}52 \\
44,44 \%\end{array}$ & $\begin{array}{c}8 \\
25,80 \%\end{array}$ & & & $\begin{array}{c}10 \\
18,51 \%\end{array}$ & $\begin{array}{c}2 \\
12,50 \%\end{array}$ & $\begin{array}{c}7 \\
12,50 \%\end{array}$ & $\begin{array}{c}2 \\
13,33 \%\end{array}$ & $\begin{array}{c}4 \\
10,25 \%\end{array}$ & $\begin{array}{c}1 \\
9,09 \%\end{array}$ \\
\hline Sandoimo & $\begin{array}{c}5 \\
4,27 \%\end{array}$ & $\begin{array}{c}1 \\
3,22 \%\end{array}$ & & & & & $\begin{array}{c}1 \\
1,78 \%\end{array}$ & & $\begin{array}{c}2 \\
5,12 \%\end{array}$ & \\
\hline Natsuimo & $\begin{array}{c}4 \\
3,41 \% \\
\end{array}$ & & $\begin{array}{c}2 \\
5,55 \% \\
\end{array}$ & & $\begin{array}{c}6 \\
11,11 \% \\
\end{array}$ & $\begin{array}{c}1 \\
6,25 \% \\
\end{array}$ & & & & \\
\hline Akiimo & & & & & $\begin{array}{c}1 \\
1,85 \%\end{array}$ & & & & & \\
\hline Gogatsuimo & & & & & & & $\begin{array}{c}6 \\
10,71 \% \\
\end{array}$ & & & \\
\hline Shinaimo & & & & & & & & & $\begin{array}{c}1 \\
2,56 \%\end{array}$ & \\
\hline Karaimo & $\begin{array}{c}1 \\
0,85 \% \\
\end{array}$ & & & & & & $\begin{array}{c}1 \\
1,78 \% \\
\end{array}$ & & & \\
\hline $\begin{array}{l}\text { Orandaimo } \\
\text { (et variantes } \\
\text { proches) }\end{array}$ & & & & & & & & & $\begin{array}{c}2 \\
5,12 \%\end{array}$ & \\
\hline $\begin{array}{l}\text { Kōboimo } \\
\text { (et variantes } \\
\text { proches) }\end{array}$ & $\begin{array}{c}1 \\
0,85 \%\end{array}$ & & & & & & $\begin{array}{c}5 \\
8,92 \%\end{array}$ & & $\begin{array}{c}1 \\
2,56 \%\end{array}$ & \\
\hline $\begin{array}{l}\text { Kōshiimo } \\
\text { (et variantes } \\
\text { proches) }\end{array}$ & $\begin{array}{c}4 \\
3,41 \%\end{array}$ & & $\begin{array}{c}1 \\
2,77 \%\end{array}$ & & & & & & $\begin{array}{c}1 \\
2,56 \%\end{array}$ & \\
\hline Autre & $\begin{array}{c}10 \\
8,54 \%\end{array}$ & $\begin{array}{c}2 \\
6,45 \%\end{array}$ & $\begin{array}{c}7 \\
19,44 \%\end{array}$ & $\begin{array}{c}3 \\
15,00 \%\end{array}$ & $\begin{array}{c}10 \\
18,51 \%\end{array}$ & $\begin{array}{c}1 \\
6,25 \%\end{array}$ & $\begin{array}{c}5 \\
8,92 \%\end{array}$ & $\begin{array}{c}1 \\
6,66 \%\end{array}$ & $\begin{array}{c}3 \\
7,69 \%\end{array}$ & \\
\hline Total & $\begin{array}{c}117 \\
100 \%\end{array}$ & $\begin{array}{c}31 \\
100 \%\end{array}$ & $\begin{array}{c}36 \\
100 \%\end{array}$ & $\begin{array}{c}20 \\
100 \%\end{array}$ & $\begin{array}{c}54 \\
100 \%\end{array}$ & $\begin{array}{c}16 \\
100 \%\end{array}$ & $\begin{array}{c}56 \\
100 \%\end{array}$ & $\begin{array}{c}15 \\
100 \%\end{array}$ & $\begin{array}{c}39 \\
100 \%\end{array}$ & $\begin{array}{c}11 \\
100 \%\end{array}$ \\
\hline
\end{tabular}

Nous observons pour les quatre premières appellations listées dans le tableau que l'emploi de la forme véhiculaire jagaimo est en hausse dans chaque préfecture. C'est notamment le cas dans la préfecture de Toyama où cette appellation remplace progressivement la forme dialectale jagataraimo qui est en fort recul. Des formes comme natsuimo qui se retrouvaient dans les préfectures de Toyama et d'Ishikawa, ainsi que sandoimo, gogatsuimo ou encore kōboimo dans celle de Fukui ont disparu au cours des cinquante dernières années.

\section{Toponymes et noms dialectaux de la pomme de terre dans la préfecture de Toyama}

\subsection{Noms dialectaux de la pomme de terre dans la préfecture de Toyama}


Carte P. - Carte linguistique des bassins des rivières Shō et Jinzū : tous les noms vernaculaires de la pomme de terre.

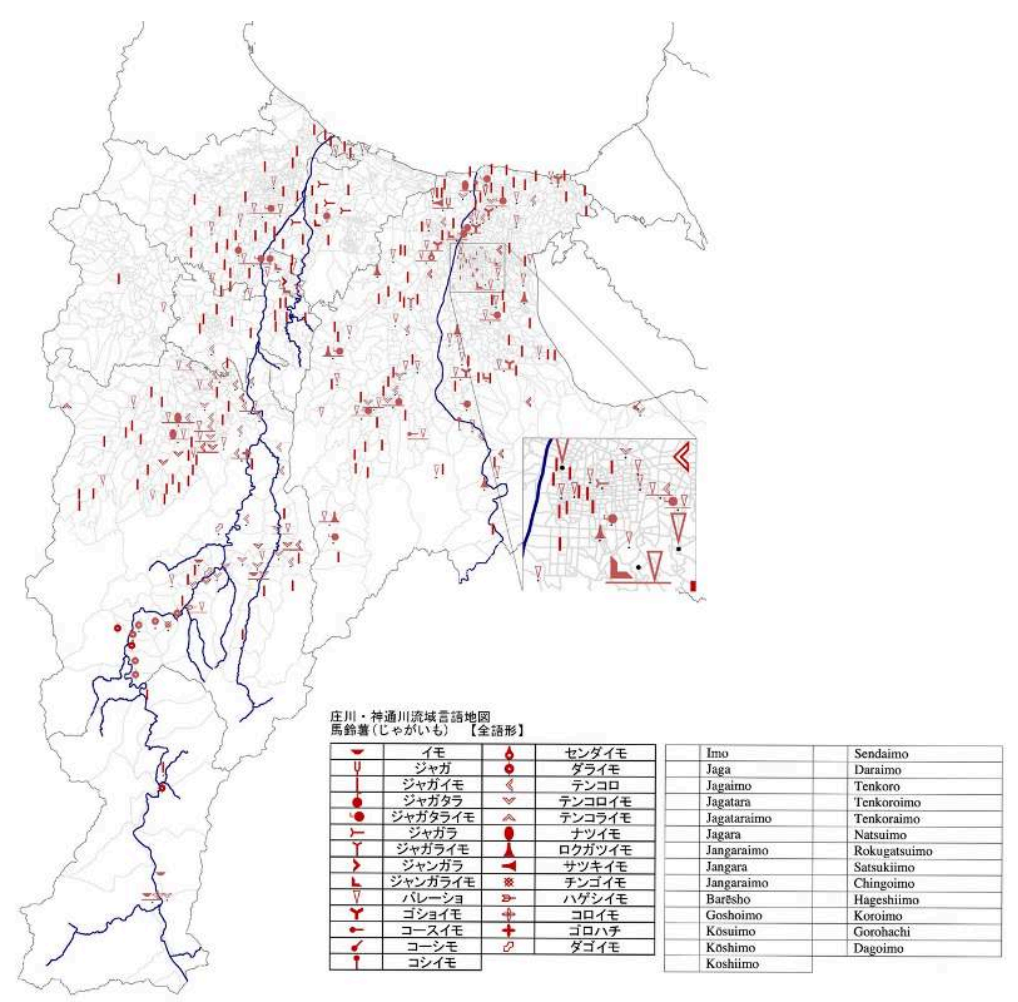

Analysons la répartition de ces différents noms dialectaux. Les formes jaga, jagaimo ou encore barēsho sont employées dans l'ensemble de la zone géographique. La réponse imo a été obtenue dans quatre points d'enquête, à savoir Kogurusu dans l'ancien village de Taira et Uebatake dans celui de Toga, tous deux situés sur le cours supérieur de la rivière Shō, ainsi qu'à Kidani et Hirase dans le village de Shirakawa, lui-même situé dans la préfecture de Gifu.

La forme daraimo est employée à Sugio (ancien village de Taira) ainsi qu'à Atarashiya, Higashiakao, Hosojima, Narude, Takasorei, Kaimukura, Shitajima et Tanoshita (ancien village de Kamitaira). Ces zones, situées sur le cours supérieur de la rivière Shō, forment la région de Kogayama. Le préfixe dara signifie 'idiot', en raison de la facilité de culture de ce tubercule qui offre deux voire trois récoltes par an.

Les formes tenkoro et tenkoroimo sont employées sur le cours moyen de la rivière Shō à Imazato, Kitaichi et Izemi dans l'ancien bourg d'Inami, à Aoshima, Kanaya et Yuyama dans celui de Shōgawa, à Takagi et Yasukiyo dans celui de Fukuno, à Uchio dans celui de Jōhana, à Yamada dans celui de Fukumitsu, à Inokuchi dans l'ancien village du même nom, ainsi que dans la vallée de Toga. Ces appellations proviendraient du maillet en bois (tenkoro) utilisé dans ces zones géographiques.

Quant aux formes telles que natsuimo employée dans l'ancien village d'Inokuchi sur le bassin de la rivière Shō et dans l'arrondissement de Kusajima à l'embouchure de la rivière Jinzū, rokugatsuimo et satsukiimo employées dans le district de Hosoiri en amont de la rivière Jinzū ainsi qu'à Yamamuro et Shinjō dans la ville de Toyama en aval, ou encore hageshiimo employée à Tamukai (ancien village de Taira), elles correspondraient toutes à des périodes de récoltes. En effet, les préfixes natsu et rokugatsu signifient respectivement 'été' et 'juin', tandis que satsuki correspond au cinquième mois du 
calendrier luni-solaire. Le préfixe hageshi se rapporterait au hangeshō, onzième jour après le solstice d'été.

\section{Relation entre toponymes et noms dialectaux de la pomme de terre dans la préfecture de Toyama}

La forme goshoimo est employée par exemple à Mizuhashi, zone située à l'embouchure de la rivière Jinzū, dans la ville de Toyama. Cette forme se rapporterait au grand rendement du tubercule, une autre hypothèse la relie aux toponymes Gosho, Kōshū et Gōshū.

La forme koshiimo, employée à Ogurosedani (ville de Toyama), se rapporte aux toponymes Koshi et Kōshū. Il est généralement admis qu'il en va de même pour les formes kōsuimo et kōshimo, employées respectivement à Kurosedani et Omi dans cette même ville.

La forme sendaimo, employée dans le district de Hosoiri sur le cours supérieur de la rivière Jinzū, semble liée au toponyme Sendai. Le professeur Ōnishi Takuichirō, affilié au NINJAL, suggère cependant que l'on doit son origine à Nakai Seidayū, et ce dans chaque région où elle est employée. Seidayū, magistrat de la ville de Kōfu, a par la suite fait parvenir cette variété depuis l'île de Kyūshū et a grandement contribué à sa transmission. Palliant aux pénuries alimentaires de l'époque grâce à ses mesures, elle prendra à certains endroits l'appellation seidayūimo. Sa culture se répandra ainsi dans tout le pays comme aliment de famine.

Carte Q. - Carte linguistique des bassins des rivières Shō et Jinzū : noms dialectaux spécifiques de la pomme de terre.

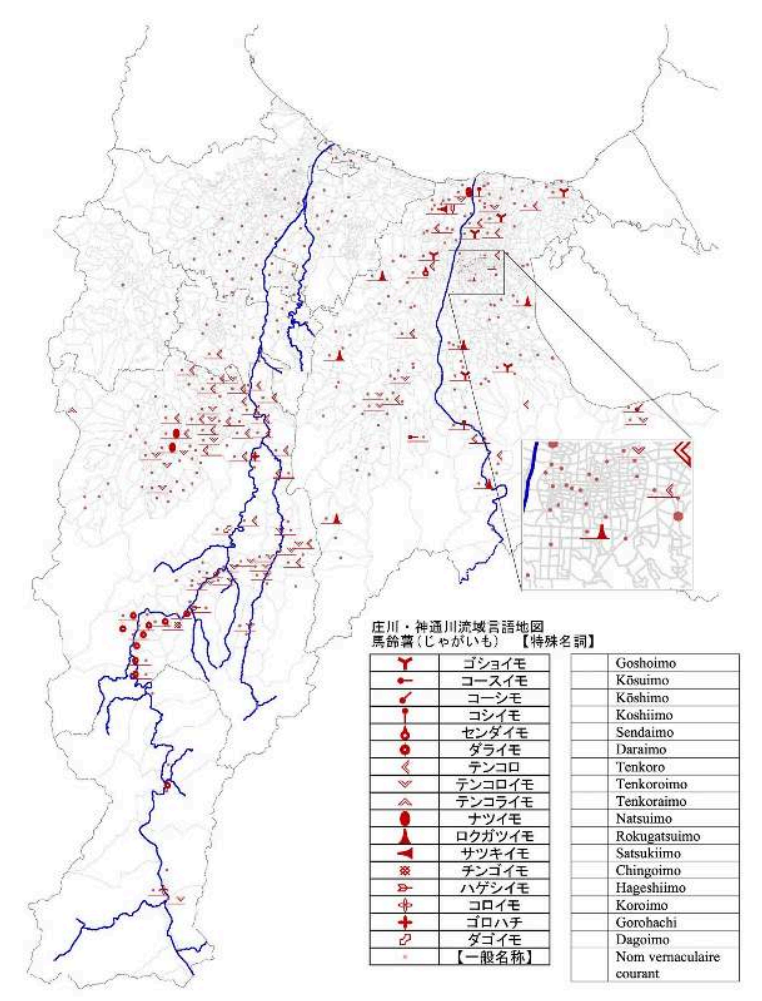


37 Alors que les bassins des rivières Shō et Jinzū s'écoulent dans la partie centrale de la préfecture de Toyama, les noms régionaux de la pomme de terre liés à des toponymes apparaissent surtout sur la rive est du bassin de la rivière Jinzū.

\section{Ce que révèlent les noms dialectaux de la pomme de terre : dénominations toponymiques et partition dialectale de la préfecture de Toyama}

Les collines de Kureha divisent la préfecture de Toyama en deux parties : Gotō ('à l'est de Kureha') et Gosei ('à l'ouest de Kureha'). Nombreux sont les habitants locaux qui emploient ces termes au quotidien. Toyama, ville emblématique de la partie est, s'étend sur le cours de la rivière Jinzū, tandis que Takaoka, sa semblable dans la partie ouest, s'étend sur le cours inférieur de la rivière Shō. Selon Mori Takashi, représentant de la Toyama minzoku no kai (Association du folklore de Toyama) ${ }^{5}$ :

Takaoka est une grande ville située dans la partie ouest de la préfecture qui appartient à l'ancien domaine de Kaga et recèle un patrimoine culturel important, qu'il s'agit de préserver.

La partie est de la préfecture étant parcourue par cinq des sept rivières de l'ancienne province d'Etchū, les inondations y ont été fréquentes. Toyama, grande ville de cette région, a de plus subi de nombreux incendies par le passé. Le grand raid aérien du $1^{\mathrm{er}}$ août 1945 constitue l'évènement le plus dévastateur, détruisant le cœur de la ville à $99,5 \%$ et n'épargnant ainsi qu'une infime partie de son patrimoine culturel. Par conséquent, forts de leur expérience en matière de reconstruction, les habitants de Gotō montrent une rapidité de décision dans tous les domaines. À l'inverse, les habitants de Gosei agissent moins rapidement en raison de leur conscience collective qui s'enracine dans une forte tradition conservatrice.

41 Dans la partie ouest, la danse du lion est effectuée en groupe et un nombre impressionnant de festivals y sont célébrés, comme le Hikiyama matsuri. Cette région est financièrement prospère et son patrimoine culturel a été entièrement préservé.

Selon les écrits de Shiba Ryōtarō dans son œuvre intitulée Kaidō o yuku (série d'essais de voyage), les collines de Kureha divisent la préfecture de Toyama entre la sphère culturelle du Kantō et celle du Kansai, chacune arborant un mode de vie, une approche commerciale et un dialecte distinct. Mori fait part de son point de vue quant au caractère de cette œuvre : « [D'après ce que j'ai lu] je pense que Monsieur Shiba n'avait peut-être pas fait suffisamment de recherches. [...] J'ai le sentiment que sa propre expérience était limitée et que son analyse de la division est-ouest ne reposait pas sur une pratique du terrain. »

Yanagita Kunio, dans son traité intitulé Minkan-denshō-ron (Théorie des traditions populaires, 1934), distingue trois catégories de documents ethnographiques :

- les «sources corporelles» qui se manifestent visuellement, à savoir toute tradition s'inscrivant dans la matérialité et l'action, qu'un voyageur pourrait observer s'il y prête un peu attention ;

- les « sources orales » que l'ouïe peut saisir, à savoir toute tradition orale qu'un emploi même minimal de la langue locale permet d'appréhender pour peu qu'on tende l'oreille ; 
- les « sources morales ", à savoir toute tradition morale, dont le sens échappera au voyageur, et qui ne sera compréhensible qu'à travers la sensibilité des habitants locaux.

Il développe son propos en classant comme suit ces trois catégories :

1. la culture matérielle et l'ethnographie des moyens d'existence, science des voyageurs ;

2. les arts langagiers et la littérature orale, science des résidents temporaires ;

3. l'interprétation, les concepts et les différentes modalités de l'existence, science des habitants locaux.

Ici, Shiba se rapporterait au point 1 et Mori au point 3.

En voyageant au cœur de la préfecture de Toyama, il n'est pas rare d'entendre parler des nombreuses inondations survenant dans Gotō, ou encore des habitants locaux de l'époque qui ont assez tôt été amenés à gagner leur vie en vendant des médicaments à base de plantes ou en partant travailler loin de chez eux. En effet, la productivité agricole de cette partie de la préfecture est faible en raison des plaines bien plus étroites que celles s'étendant dans Gosei.

Notons que les noms dialectaux de la pomme de terre se répartissent de manière cohérente depuis le cours supérieur de la rivière Shō, dans la partie ouest, comme suit : imo $\rightarrow$ daraimo $\rightarrow$ tenkoro ou tenkoroimo $\rightarrow$ jagaimo et bareisho (noms prévalents). Dans le bassin de la rivière Jinzū situé dans la partie est, en revanche, nous remarquons l'emploi de formes associées à des périodes de récoltes telles que natsuimo, rokugatsuimo ou encore satsukiimo. De plus, certaines formes révèlent des toponymes comme goshoimo (Gosho, Kōshū, Gōshū), kōsuimo et koshiimo (Koshi, Kōshū). Chacun de ces deux bassins présente ainsi des aspects bien distincts. Enfin, chaque nom dialectal révélant un toponyme est associé à Nakai Seidaiyū, magistrat de $\mathrm{Ko}^{\mathrm{f}} \mathrm{u}^{6}$, et permettrait de retracer l'itinéraire par lequel la pomme de terre s'est transmise sur la rive est du bassin de la rivière Jinzū.

La pomme de terre et la patate douce se sont toutes deux répandues au Japon comme aliments de famine. Nous constatons pour la seconde que les noms dialectaux motivés par un toponyme connaissent une grande aire d'extension: karaimo, ryūkyūimo puis satsumaimo. Ce n'est pas tant le cas de la première; fait qui s'explique probablement par les modalités et les périodes de propagation, ainsi que par les procédés de culture et d'utilisation. En analysant les toponymes associés aux noms vernaculaires de cultures agricoles, il nous est possible de découvrir des spécificités historiques et socioculturelles des régions qui nous échappaient jusqu'alors.

\section{BIBLIOGRAPHIE}

KATō Masanobu, SATō Takeyoshi \& MAEDA Tomiyoshi, 1988, Hōgen ni ikiru kogo, Nan'undō.

KōKURITSU KoKUGo Kenkyūjo, 1984, Nihon gengo chizu kaisetsu: kaku zu no setsumei 4 / Introduction to The Linguistic Atlas of Japan: Interpretation of the Maps, vol. 4.

MAKINo Ryūshin, 1979, Kitamaebune no jidai: kindai igo no nihon-kai kaiunshi, Kyōikusha. 
MuконARA Yoshitaka, 2004, «Shin Satsumagaku: Satsuma, Amami, Ryūkyū », dans Nanpō shinsha Satsuma, p. 26-29.

NAKAI Seiichi, 2011, « Gendai hōgen kara mita shokubutsu riyō no chiiki tayōsei », dans Shirīzu Nihon-rettō no san man go sen nen: hito to shizen no kankyōshi - Dai roku kan: kankyōshi o toraeru gihō, Bun-ichi Sōgō Shuppan.

NōSANGYOSON Bunka Kyōkai, 1975, Nōgyō gijutsu taikei sakumotsu hen 5: jagaimo, satsumaimo, p. 15-22.

SAKOGUCHI Yukako \& KISHIE Shinsuke, 2013, Awaji-shima no hōgen goi ni kansuru kenkyū: jagaimo, satsumaimo, satoimo / A Study of Geographical Dialect in Awaji Island: Potato, Sweet Potato, Taro, Gengo bunka kenkyū.

SATō Ryōichi, 1982, « Hōgen goi no punpu: "Nihon gengo chizu" ni miru », dans Kōza nihongo no goi 8: gengo no goi, Meiji Shoin.

SAWAKI Motoei, 1979, « Mono to kotoba », dans Nihon no gengo chizu, Chūkō Shinsho.

SHIBA Ryōtarō, 1974, Kaidō o yuku (4), [Éditions] Asahi shinbun shuppan-kyoku, 398 p.

TAGOMORI Hiroshi, 1992, « Hōgen goi shiryō toshite no shokoku sanbutsu-chō: "kansho" to "kabocha" o chūshin ni / Use of The Product Registers of Various Prefectures (Shokoku Sanbutsu-chō) as Dialect Vocabulary Data: Focusing on Words for "Sweet Potato" and "Pumpkin" ", dans Kokugo gaku, nº 171.

YANAGITA Kunio, 1998, « Minkan denshō ron » (Une théorie des traditions populaires) [1934], dans Id., Yanagita Kunio zenshū (๕uvres complètes de Yanagita Kunio), Tokyo, Chikuma shobō, vol. 8.

\section{NOTES}

1. Satsumaimo, nom vernaculaire le plus communément attribué à la patate douce.

2. Jagaimo, nom vernaculaire le plus communément attribué à la pomme de terre.

3. Jagataraimo, soit 'tubercule de Jakarta' à partir d'une prononciation régionale du toponyme.

4. Ezoimo, soit 'tubercule d'Ezo', ancien nom de Hokkaidō.

5. Selon les propos recueillis par l'auteur lors d'une discussion le 11 octobre 2019 avec Mori Takashi.

6. Kōfu et Kōshū sont des villes voisines. La pomme de terre est ainsi appelée 'tubercule de Kōshū (Gōshū, Koshi, etc.)' en lien avec le magistrat Nakai Seidayū, originaire de cette région.

\section{RÉSUMÉS}

L'objectif de cet article est de montrer que les multiples appellations japonaises de la patate douce et de la pomme de terre sont liées à des toponymes, des périodes de récoltes ou encore des spécificités dialectales. Il présente également l'évolution dans le temps de l'emploi des appellations de la pomme de terre dans tout l'archipel japonais, et s'articule enfin plus particulièrement autour des noms dialectaux de la pomme de terre dans la préfecture de 
Toyama. L'auteur s'appuie sur le Linguistic Atlas of Japan ainsi que sur le New Linguistic Atlas of Japan pour exposer ces résultats.

The aim of this paper is to show that the many Japanese names for sweet potato and potato are linked to toponyms, harvest periods or dialectal specificities. It also presents the evolution over time of the use of potato names throughout the Japanese archipelago, and finally focuses more specifically on the dialectal names of potatoes in Toyama Prefecture. The author relies on the Linguistic Atlas of Japan and the New Linguistic Atlas of Japan to show these results.

INDEX

Keywords : names, toponyms, potato, sweet potato, Toyama, Japan

Mots-clés : appellations, toponymes, pomme de terre, patate douce, Toyama, Japon

\section{AUTEURS}

\section{SEIICHI NAKAI}

Université Dôshisha, département des arts libéraux

Doshisha Women's College of Liberal Arts

s-nakai@dwc.doshisha.ac.jp 